\title{
AASMAn: An adaptive organisational policy for a society of market-based agents
}

\author{
$S_{S}$ SHAHEEN FATIMA ${ }^{1}$ and $\mathrm{G} \mathrm{UMA}^{2}$ \\ ${ }^{1}$ Department of Computer Science \& Engineering, M J College of Engineer- \\ ing, Rd. No. 3, Banjara Hills, Hyderabad 500034 , India \\ ${ }^{2}$ D E Shaw India Software Pvt Ltd, G Pullareddy Building, Begumpet, \\ Hyderabad 500016, India \\ e-mail: sshaheenfatima@hotmail.com;guma@deshaw.com
}

MS received 11 June 1997; revised 9 December 1997

\begin{abstract}
Several formalisms for implementing organisational policies that assign specific roles to each of the agents in a multi-agent system (MAS) have been proposed like the contract net protocol, the social reasoning mechanism and the distributed computational economy. However, as no single organisation is appropriate in all situations, organisation self design had been proposed to allow an organisation of problem solvers to adapt itself to a dynamically changing environment. In this paper we propose an adaptive organisational framework that exploits and integrates the essential features of these existing organisational policies not only to meet the needs of time constrained noncritical applications, where computational load on the organisation cannot be predicted ahead of time, but also to utilise the available system resources efficiently. We also introduce the notion of decommitments and penalties in this approach which was not present in the original contract net protocol.
\end{abstract}

Keywords. Multi-agent system; contract net protocol; social reasoning mechanism; distributed computational economy; organisation self design.

\section{Introduction}

Artificial intelligence is the study of computations that make it possible to perceive, reason and act. Distributed artificial intelligence (DAI) systems were developed as a sequel to earlier AI systems which were stand-alone systems that solved problems with minimal help from other systems (human or computer). But these systems failed miserably when presented with problems even slightly outside their limited range of expertise. The strategy of injecting more knowledge into the system to enlarge the systems' range of capabilities provided only a short-term answer to the problem. A more powerful strategy which has been developed puts the system into a society of systems where it can draw on the expertise and 
capabilities of other systems in the society. The overall system then becomes a collection of individual modules which interact productively to solve a problem. Each individual module is an intelligent agent and the whole system is called a multi-agent system (MAS). A recent special issue of the Communications of the ACM (Reicken 1994) is devoted to this topic.

In a MAS no single agent has the complete knowledge to solve a problem independently. The entire knowledge is split across several agents. In order to solve a problem, an agent in a MAS divides it into subproblems and allocates these subproblems to suitable partner agents. These agents solve the allocated subproblems and report results to the allocating agent which then integrates all partial results into a complete solution. The goal of MAS design is therefore to create a team of co-operating agents that share their knowledge and skills to solve even complex problems very easily.

Multi-agent systems are primarily applicable to domains like sensing and control of air or ship traffic that involve tasks such as detection, classification and tracking of vehicles to obtain a map of the traffic in some area. Construction and maintenance of the map requires interpretation of sensory information received by a collection of sensory elements that are spread over a large geographic area. The solution can be developed as a MAS where each agent has either sensing or processing capabilities. Other domains like power plant equipment monitoring systems which require tasks such as condition monitoring, fault detection, diagnosis and treatment to be performed continuously can also be easily developed as a MAS. In general, MAS find application in areas where tasks to be performed are inherently distributed and the skills required to carry out these tasks are split among several agents. The advantages of applications developed as MAS are two-fold: increased efficiency and increased capabilities.

MAS research is concerned with co-ordinating intelligent behaviour among a collection of autonomous intelligent agents, i.e. how they co-ordinate their knowledge, goals, skills and plans jointly to take action or to solve problems. Agents can define their own goals and plans and can perform complex interactions with other agents. They are independent of any particular problem-solving organisation, they can define and change the organisation as the problem-solving activity goes on. This is possible only when agents are adaptive in nature. The agents need to adapt themselves to changes in the environment, for instance by choosing different goals to achieve, plans to execute or partners with whom to work cooperatively depending upon the available services. Most situations consist of a collection of agents with various skills including sensing, communication, planning and acting. Such group situations possess the following characteristics.

Openness: Agents enter and leave the society at any time.

Autonomy: Individual agents decide whether to co-operate with other agents or not. Groups of agents that decide to co-operate and work towards a common goal are said to form a coalition.

Adaptability: Agents need to adapt themselves to changing environmental demands.

Limited knowledge: An agent may be subject to several kinds of limitations like limited knowledge of environment, limited knowledge of the tasks ${ }^{1}$ of the group or of the intentions of other agents.

1'The words 'goal', 'task' and 'problem' have been used interchangeably 
Shared limited resources: There are often shared limited resources with which each agent can attack tasks.

Differing agent capabilities: Agents typically have differing appropriateness for a given task. The appropriateness of an agent for a task is a function of how well the agents' skills match the expertise required to do the task, the extent to which its limited knowledge is adequate for the task and the current processing resources of the agent.

Several kinds of distributed problem-solving difficulties accrue from this characterisation. First there are difficulties with 'optimal task assignments'. Many mappings of decomposed sub-tasks to agents may be possible but, because agents have differing appropriateness with respect to a given task, only a few agents will be acceptable for any task. It is therefore crucial for agents to adopt the right role. Second, 'task co-ordination problems' arise because tasks assigned to agents may not be independent. In summary, the main challenge to multi-agent systems is that the solutions that an agent produces must not only be locally acceptable, and achieve the assigned goals, but must also be interfaced correctly with the actions of other agents solving dependent tasks. Similarly, the solutions must not only be reasonable with respect to the local task but must also be globally coherent, and this global coherence must be achieved by local computations alone. These difficulties can be overcome by choosing effective strategies for co-operation and communication. Two distinct classes of such strategies are 'organisational policies' and 'information distribution policies'.

Organisational policies dictate how sub-tasks of a large task should be allocated to individual agents. These policies prescribe communication paths among agents. They turn a random collection of agents into a network that is fixed at least for a given task. Decisions about how agents communicate with each other are constrained by the choice of organisational policy, since the policy decides the network of permissible communications. However, within these constraints, a large number of lower level decisions must be made about how and when communications should occur. Information distribution policy determines these lower level decisions.

The key issue in multi-agent system research is therefore that of developing effective and adaptive organisational policies that determine how goals are to be distributed among agents. There must be a means whereby agents with goals to be achieved can find the most appropriate idle agents to achieve those goals (Cammarata et al 1983; Durfee et al 1985; Decker 1987).

Several formalisms for implementing organisational policies have been proposed. In $\S 2$ we briefly examine these mechanisms, namely Contract Net Protocol (CNP), Social Reasoning Mechanism (SRM), and Distributed Computational Economy (DCE). We find that CNP, SRM and DCE have each taken one aspect of problem solving in human societies and put forward a computational model for that. Organisation Self Design (OSD) is targeted to achieve load balancing in a distributed environment. But since co-ordination in human societies is based on many aspects including dependence, material benefits and social laws, we believe that we could build open multi-agent systems that are closer to human societies by developing a mechanism to implement all these characteristics (Fatima \& Uma 1998). In $\S 3$ we propose a framework that combines all these aspects to obtain a new organisational policy for open MAS that is suited to time constrained domains. Section 4 gives the results 
of simulation of this model. Finally we show empirically that our proposed model has the characteristics of adaptiveness, meeting deadlines and effective resource utilisation, and also highlight the direction for further work.

\section{Related work}

\subsection{Contract net protocol}

The primary goal of CNP (Smith 1980; Smith et al 1981; Davis \& Smith 1983; Cheng \& Ishidà 1996) is opportunistic and adaptive task allocation among a collection of problem solvers using a framework called negotiation based on task announcements, bids and awarded contracts.

Two kinds of agents, 'manager' and 'contractor' exist in CNP. Managers provide tasks to contractors which undertake them as follows: First, when a task arrives at the system, the manager responsible for it announces it to all contractors within the system; second, each contractor selects from the announced tasks the one that best matches its own capabilities and bids for it; finally, the manager chooses what it believes to be the most appropriate bid and awards the task to that agent. There may exist multiple managers and contractors in the system simultaneously; a manager can make multiple announcements simultaneously, while a contractor can bid for only one task at a time. The most significant feature of CNP is that both managers and contractors award or bid according to their own standards. This mechanism is called negotiation or mutual selection.

In the case of CNP, agents are assumed to provide their services without any motivation. However, this situation can be modified by having the manager announce some rewards at the time of task announcement which can serve as a motivation for dynamic coalition formation.

\subsection{Social reasoning mechanism}

In the SRM model (Sichman \& Demazeau 1995) dynamic coalitions are formed on the basis of motivation in the form of social dependencies. Agents provide services to other agents that request them, only if they believe that they can obtain some other service in return from the requesting agent, i.e. they have a social dependence relation with each other. Agents are assumed to have information about the other agents attributes (capabilities, reasoning mechanisms, restrictions, performance etc.). Using this information, an agent infers its dependence relation with other agents. An agent is said to be autonomous for a given goal if it has the capability to achieve the goal independently. Three types of dependence relations are defined: mutual dependence (MD), reciprocal dependence (RD) and unilateral dependence (UD). A MD is said to exist between two agents if they depend on each other for the same goal. RD exists between two agents if they depend on each other for different goals: When an agent depends on another agent for a goal but the latter agent does not depend on the former agent for any of its goals then the former agent is said to be unilaterally dependent on the latter agent. These dependence relations are used to establish some decision criteria in the form of social laws to help an agent choose its partners when it is not able to achieve a goal alone: It is always best for an agent to choose as partner 
another agent with whom an MD relation exists because in this case the other agent would definitely co-operate. A second best choice is to select a partner with whom an RD exists, since in this case the agent can propose an exchange (if you help me to achieve my goal, I'll help you to achieve yours). A UD is the worst choice since the agent has nothing to offer in return.

\subsection{Distributed computational economy}

The similarity between task allocation problems of MAS and economics resulted in the application of markets to MAS. Numerous projects have applied market mechanisms to problems in distributed task allocation (Waldspurger et al 1992). In this approach, agents are participants in a computational economy, interacting in the market to further their own interests. System behaviour is described in standard economic terms of buying, selling, auctions, bidding, funds, profits and exchange. To formulate a problem as a computational economy, the activities of interest are cast in terms of buying and selling of services and agents are designed to choose strategies for buying and selling based on their own capabilities and preferences and the going market price for services. Such environments are expected to become increasingly common for MAS because the 'market' is the world standard default interface for interacting entities. A more complete description of market agents is given in $\S 3$.

\subsection{Organisation self design}

These are some of the approaches to organisation. However as no single organisation is appropriate in all situations, techniques for building problem solving systems that can adapt to changing environmental conditions are of great interest. Towards this end OSD was proposed to allow an organisation of problem solvers to adapt itself to dynamically changing situations. Ishida and others (Ishida et al 1992; Ishida 1995) present an approach that relies on the reorganisation of a collection of problem solvers to track changes in response requirements. Their approach exploits an adaptive allocation of resources and organisation forms to satisfy performance constraints. Agents are created and released and domain knowledge is continually reallocated. To extend the agent architecture for OSD they introduce composition and decomposition of agents as new reorganisation primitives. They also formalise organisational knowledge to represent interactions among agents and their organisation. The details of composition and decomposition primitives are given in $\S 3 c$.

All these organisational policies were developed by incorporating important problemsolving mechanisms of human societies where multiple parties are responsible for doing a single task. Overall, each of these developments provides a rich ground for the development of concepts and implementation of one aspect of human organisation in MAS. But in human societies, each of these aspects is not considered in isolation. At any point of problem-solving, one or more of them may play a role in reasoning. Towards this end we present a protocol that exploits the negotiation mechanism of the CNP, social laws of the SRM, market economy feature of the DCE and OSD, and integrates these features to meet the needs of time-constrained domains where the computational load on the organisation 
cannot be predicted ahead of time. We also introduce the notion of decommitments and penalties in this approach, which was not present in the original CNP.

\section{AASMAn: An adaptive organisational policy for a society of market-based agents}

In this framework multiple agents co-operate to solve a single or multiple problems. At a high level of description, agents are organised as a market economy composed of interacting buyers and sellers. The commodities in the market are skills or services associated with the agents. Buyers are agents who wish to purchase skills in order to achieve goals within the constraints of deadlines and resources. Sellers are agents that wish to sell their skills because they are idle. The resources can be quantified using the notion of funds.

The basic architecture comprises agents with manager and contractor roles. The manager agent is initially assigned a task and is also allocated some funds. The manager has to efficiently utilise these funds to accomplish the assigned task within the specified deadline. The manager decomposes the task into sub-tasks. If the manager finds that it can perform all the sub-tasks within the stipulated time then it does so. Otherwise, it forms a coalition with the other agents in the society called contractors and all agents in the coalition then work together towards achieving the goal. On completion of the assigned sub-task the contractors send partial results to the manager which then transfers funds to the contractors and integrates all these partial results into a complete solution. Contractors for coalition formation are chosen based on goal deadline, funds allocated to the manager and the current market price for buying a service.

Idle agents which have no goals/tasks at hand make auctions for other agents to buy their skills. This is known as selling of services. These auctions specify the available skills, time requirements and the initial price. These agents are known as seller agents. As agents are assumed to have differing capabilities the initial price for a service is fixed depending on the agent's capability, i.e. its speed, reliability etc. However, this price changes when the service being provided is in demand and there are many agents bidding for it. In such a situation manager agents offer a higher price to buy the service since the bid with the highest price is given the highest priority.

The manager negotiates with other agents to select contractors for coalition formation. The aim of a manager in need of forming a coalition is to efficiently utilise funds within the constraint of meeting deadlines. It therefore listens to these auctions, determines the most suitable contractors for coalition formation and then sends bids to these agents to buy the required services.

A seller may receive more than one bid for a single service but the bid that offers the highest price is selected by the seller. In case there is more than one agent offering the highest price, the highest priority bid is selected. Bids are prioritised on the basis of the social dependence relation that exists with the bidding agent. The highest priority is given to an MD relation; which is followed by an RD relation; while a UD relation is given the least priority. The seller chooses the bid with the highest priority and sends a commitment message to the agent that sent the bid. The seller now becomes the contractor for the requested goal. 
If the manager receives the commitment message, a coalition of buyers and sellers is formed. Otherwise the manager agent, again on the basis of auctions, selects the next most appropriate agents for coalition formation and sends bids to them. This repeats till successful coalition formation. If coalition formation is not successful, then agent decomposition is called for (the concept of composition and decomposition is explained later in this section).

We therefore have a MAS where agents are market-based. Fundamentally, a market agent is no different from any other agent. But a key distinguishing feature of a market agent is its interface with the rest of the system. Its interactions with the rest of the system are primarily through the exchange of services and its communications are primarily devoted to arranging such exchanges. There are three major tasks specific to the economic environment which must be addressed in the design of market agents. The first task of the market agent is to identify the services available in the system so that it knows in what terms to interact with other agents. Second, every agent must solve its own optimisation problem, i.e. once it knows the services and the possible terms by which they must be exchanged, it needs to determine its exchange policy. This exchange policy is formulated to optimise some criterion like efficient utilisation of funds and maximisation of profits subject to the constraints of resource availability and deadlines. Finally the market agent must aim to implement its optimal policy through negotiating terms. This entails managing its bidding activity, and determining how best to arrange its requests and commitments to other agents to solve the optimisation problem.

To perform problem solving by multiple agents, in addition to domain knowledge, each agent needs organisational knowledge which represents necessary interactions among agents. The agent architecture describes all the knowledge that agents need to possess in order to work together as a group.

\subsection{Agent architecture}

We view an agent as having five major functional components: a sensor for sensing the environment, a communication processor that handles message traffic with other agents, a goal processor that carries out the computation associated with goals, a contract processor that actually processes different types of messages and manages agent resources, and a local knowledge base comprising the following.

Domain knowledge: This is the knowledge required for problem solving. It consists of goals and the associated plans for achieving those goals.

Organisational knowledge: In MAS, multiple agents solve a single problem or multiple problems. Interference exists among goals when the result of parallel execution of the goals is different from the results of sequential execution applied in any order. If two interfering goals are distributed to different agents, the agents have to locally synchronise to prevent the goals from being executed in parallel, and thus maintain consistency. This inter-agent inconsistency can be handled locally by using temporary synchronisation via goal-deactivation. Organisational knowledge represents the goal-dependence relationships among agents. An agent that has such a relationship with another agent is called the agents neighbour. 
The capabilities of other agents in the MAS are also stored here. This knowledge is used in determining the social dependence relation with other agents.

Priority queues and counters: Problem-solving requests arrive at an agent from two different sources: User requests ${ }^{2}$ and requests from other agents for coalition formation. These requests are stored in four separate queues $-\mathrm{Q} 1, \mathrm{Q} 2, \mathrm{Q} 3$ and $\mathrm{Q} 4$. $\mathrm{Q} 1$ stores user requests, $\mathrm{Q} 2$ stores requests from other agents with whom an $\mathrm{MD}$ relation exists, $\mathrm{Q} 3$ stores requests from other agents with whom an RD relation exists and Q4 stores requests from all other agents. The queues in the decreasing order of priorities are Q1, Q2, Q3, Q4. Apart from priority queues every agent maintains counters for counting the number of requests that arrive for every goal (Cgi).

We now introduce the goal execution protocol.

\subsection{Goal execution protocol}

The goal/task execution protocol therefore becomes

(1) Process messages: The different messages that can be received by an agent and how they are processed are listed below.

- Goal execution request message: If it is a user request and its deadline can be met independently then the request is queued in Q1, otherwise coalition formation is initiated. If the request is from any other agent and the deadline can be met, then the request is queued in $\mathrm{Q} 2, \mathrm{Q} 3$ or $\mathrm{Q} 4$ and a committment message is sent to the requesting agent, otherwise coalition formation is initiated. In both cases, if coalition formation is not successful, decomposition is initiated (decomposition is explained in § 3.3). If decomposition is also not possible, it means that the request cannot be honoured. If the request that cannot be honoured is from some other agent, it is simply ignored. However if the request is a user request then the agent selects the least priority goal (out of Q2, Q3 and Q4) for decommittment and sends a decommittment message to the manager of that goal and also pays some funds (which might be based on parameters like time spent by the task in queue, how much the agent gains in the new committment etc.) as penalty to the manager. Goals in $\mathrm{Q} 1$ are never allowed to be decommitted. The decommittment of goals continues till the new request becomes processable within its deadline.

- Synchronisation request message: Goals mentioned in the synchronisation request message are deactivated and an acknowledgement is sent to the requesting agent.

- Synchronisation release message: Goals that were earlier deactivated due to synchronisation request message are now reactivated.

- Partial results from contractors: Transfer of funds to contractor and integration of partial results takes place.

- Auctions: The most appropriate agent for coalition formation is found and a bid is sent to it.

${ }^{2}$ Information received through sensors is termed as user request 
- Bids: Bids are prioritised and a committment message is sent to the highest priority bidding agent.

- Committment: A goal award message is sent to the agent from whom the committment message is received.

- Award goal: Goal is stored in Q2, Q3 or Q4 depending on the type of agent awarding the goal.

- Decommit: The decommitted goal is treated as any other goal execution request message.

(2) Select task for execution: Highest priority task is selected for execution, $(\mathrm{Q} 1>\mathrm{Q} 2>\mathrm{Q} 3$ $>$ Q4).

(3) Request synchronisation: Using knowledge from goal dependence relations, synchronisation request messages are sent to the agents that require synchronisation. An acknowledgement is then awaited from all these agents.

(4) Execute task: The selected task is executed and results are reported to the manager.

(5) Release synchronisation: A synchronisation release message is sent to all synchronised agents.

(6) Go to step 1.

We now introduce composition and decomposition primitives of OSD.

Problem-solving requests issued from the environment arrive at the organisation continuously and at variable rates. To respond, the organisation must adapt to changing conditions and supply meaningful results within pre-specified time limits. The OSD approach relies on the reorganisation of a collection of agents to track changes in response requirements. This approach exploits an adaptive allocation of resources and organisation form to satisfy performance constraints. Agents are created and released and domain knowledge is continually reallocated. Composition and decomposition primitives are used to create and release agents. Agent composition and decomposition can occur concurrently in different parts of the organisation. Through repeated application of these primitives OSD can be locally and asynchronously performed.

Both composition and decomposition dynamically change the size of the agent population, the resources allocated to each agent and the distribution of problem-solving and organisational knowledge in the organisation. This is shown in figure 1 . In general decomposition increases the overall level of resources used while composition decreases the level of resources used. Our simulation results show the effectiveness of this approach in our model.

OSD is performed in the following way: Upon initiation only one agent containing all domain and organisational knowledge exists in the organisation. As time progresses agents keep entering and leaving the society. The following subsection introduces composition and decomposition primitives.

\subsection{Reorganisation primitives}

For effective reorganisation, agents should invoke the following reorganisation primitives. 
Problem solving requests arrive at variable rates

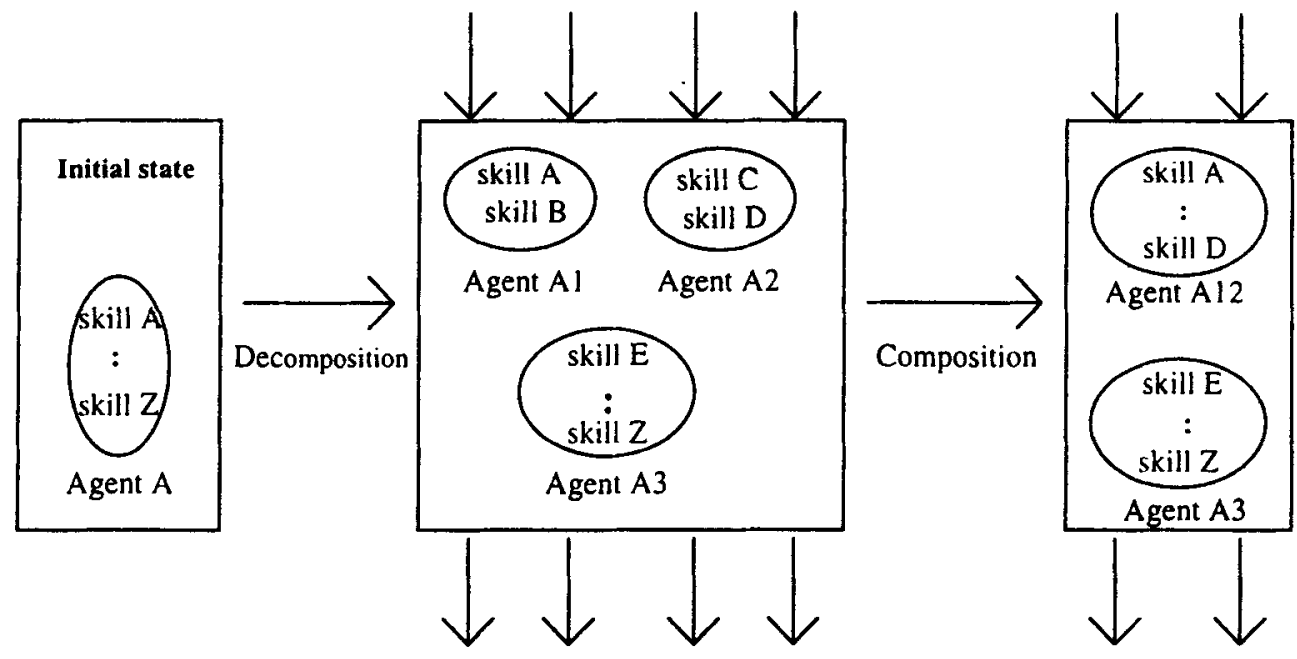

Results are required within a pre-defined time limit

Figure 1. Organisation self design.

Decomposition: Divides one agent into two. This is done either to increase intra-problem parallelism or inter-problem parallelism.

Composition: Combines two agents into one and decreases the level of parallelism. This is done either to adaptively free computing resources for cost-effective problem-solving or to reduce response time. Composition may actually reduce response time even though parallelism decreases where co-ordination overhead (i.e. communication and synchronisation overhead) is high. These are shown in figure 1.

The conditions for triggering these primitives are as follows. Decomposition is triggered when environmental conditions like problem-solving demand on the organisation and response time exceed the organisation's ability to respond, given the current form and resource level. Excessive demand at the organisation level is translated into excessive local demand in particular regions of the organisation that is measured using local and organisational statistics. At this point agents with excessive local demand are divided into multiple agents and additional computational resources are assigned to them. Decomposition continues until parallelism increases and response improves.

Composition is performed when under-utilised resources can be released for use by other agents or to improve local performance by reducing co-ordination overhead. When two agents taken together contain an oversupply of resources, they are combined into one agent via composition. Composition repeats until no more composition is possible under the conditions of meeting deadlines.

Since the aims of composition and decomposition are independent, both kinds of reorganisations can be performed simultaneously in different parts of the organisation. In this way both problem-solving and organisation self-design are treated as decentralised processes. 
Organisational knowledge is extended to include local statistics as well as organisational statistics, which are used to arrive at conditions for triggering composition and decomposition.

Local statistics: The number of entries in the queues Q1, Q2, Q3 and Q4 represent the level of activity of each agent. When this number exceeds a certain threshold value it means that the rate of arrival of problem-solving requests is very high and there are chances of a queue overflow. Thus one of the conditions that triggers decomposition is the number of entries in Q1, Q2, Q3 and Q4. A second condition for triggering decomposition is the number of problem-solving requests that are not honoured (because deadlines associated with these requests cannot be met) during a certain time period.

When Q1 is empty and the number of entries in Q2, Q3 and Q4 is less than some threshold value, it means that the agent has a very low level of activity and therefore starts making auctions for selling services to other agents. But if it does not receive any bids in response to the auction, then it means that there is no demand for the services being provided by that agent. Such a situation calls for agent composition.

Organisational statistics: It is assumed that each agent knows whether the organisation is currently meeting deadlines by periodically broadcasting messages. Let $T_{\text {complete }}$ be the expected completion time of the most recently made request and $T_{\text {deadline }}$ be the pre-defined time limit of that goal. When $T_{\text {complete }}>T_{\text {deadline, }}$, the performance of the organisation should be improved by doing a decomposition, while when $T_{\text {complete }}<T_{\text {deadline, }}$, the organisation can release resources by doing a composition.

These primitives are used in the reorganisation protocol described below.

\subsection{Reorganisation protocols}

Reorganisation is done by the execution of reorganisation tasks during the goal execution cycle. OSD and domain problem-solving are interleaved. Higher priority is given to reorganisation tasks during step 2 of the goal execution protocol. When the conditions for composition and decomposition are satisfied, the reorganisation task is selected during step 2 of the goal execution cycle. The protocol given in figure 2 describes how one agent, say A, decomposes itself into two agents, say A and B. During reorganisation, domain knowledge and organisational knowledge are transferred from agent $\mathbf{A}$ to agent $\mathbf{B}$. Information about the creation of the new agent is broadcast to all other agents in the society. All agents can then update their organisational knowledge. These steps are described below.

Decomposition protocol: (1) Create a new agent: Agent A creates a new agent, agent B, which starts task execution cycles.

(2) Select domain knowledge to be transferred to the new agent: Agent A selects the domain knowledge to be transferred to agent $\mathrm{B}$. Domain knowledge for $N / 2$ most frequently requested goals (determined from the counters) is selected for transfer to the new agent (where $N=$ the total number of goals that the agent can achieve).

(3) Request synchronisation: Agent A sends a synchronisation request message for each goal to be synchronised to agent B. Agent A also sends synchronisation request messages 


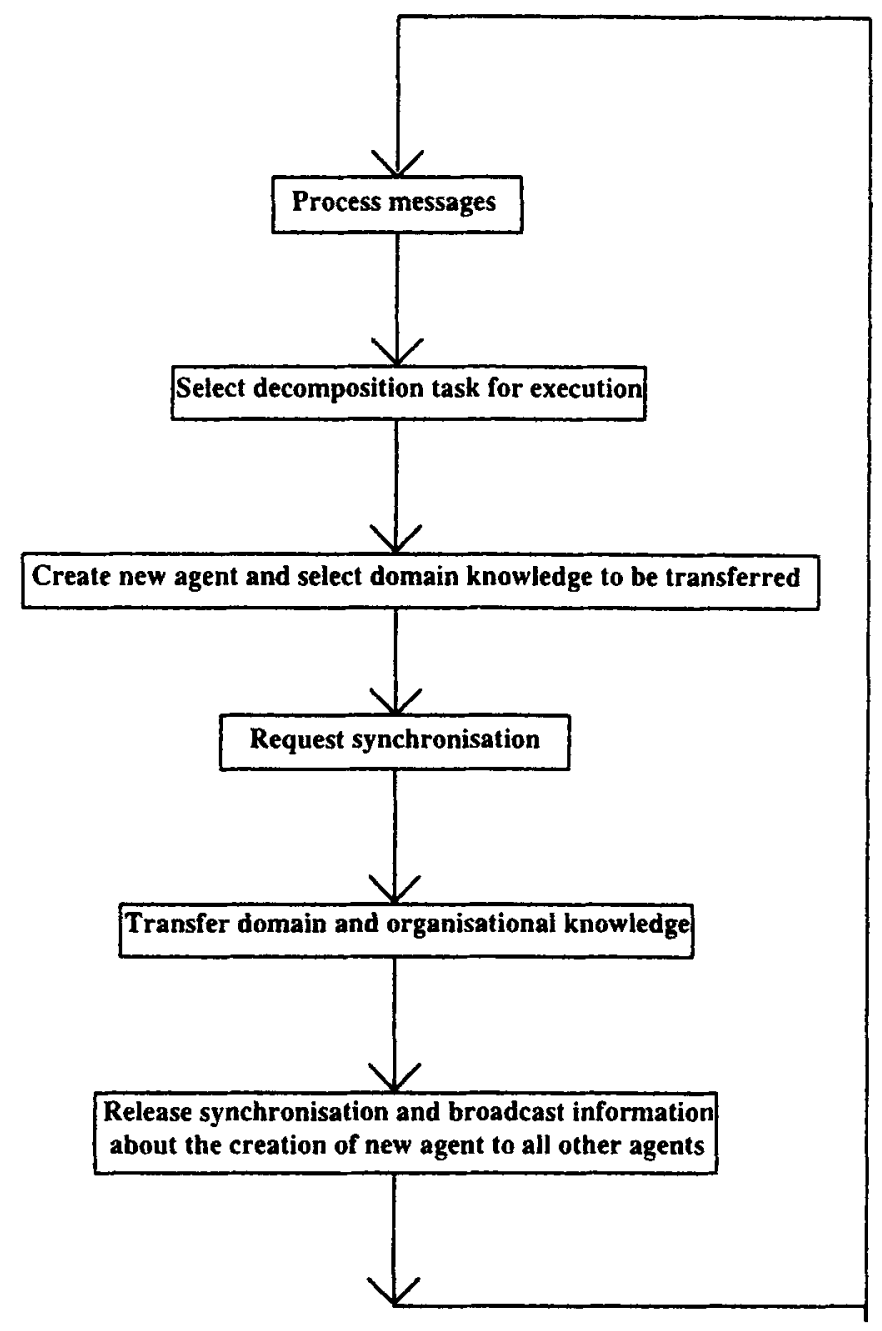

Figure 2. Reorganisation protocol.

to its neighbours. Agent A then waits for acknowledgements from all its neighbours. While waiting for acknowledgement, agent $A$ can process messages as in step 1 of the goal execution protocol.

(4) Transfer domain knowledge and organisational knowledge: Agent A transfers domain and organisational knowledge to agent $B$, updates its own organisational knowledge and also propagates changes to other agents.

(5) Release synchronisation: Agent A sends synchronisation release message to agent B and its neighbours.

This ends reorganisation. These steps are incorporated in the goal execution protocol as shown in figure 2.

Composition protocol: An agent can compose with another agent by a similar process. First agent $\mathrm{A}$ sends composition request messages to its neighbours. If some agent, say agent 
B, acknowledges, agent A transfers all domain and organisational knowledge to agent B and leaves the organisation. The transfer method is the same as that for decomposition.

\section{Simulation results}

To evaluate the effectiveness of the proposed approach a simulation environment is implemented. Experiment begins with one agent, agent 0 , which has the capability to execute 10 different goals, $g 1$ to $g 10$. The domain knowledge required to execute these 10 goals is fully available with agent 0 . Table 1 lists the time and funds required for processing each of these goals. In order to simplify the discussion we assume that all agents have equal processing speed and that all agents charge equally for the same service.

To start with, agent 0 does not have any organisational knowledge as it is the only agent in the society. However this organisational knowledge keeps changing as agents enter and leave the society.

In the beginning all goal processing requests arrive at this agent. As time progresses, agents keep entering and leaving the society, But at any point of time every agent in the society exhibits similar behaviour under similar environmental conditions. We therefore describe in detail the behaviour of just one agent, agent 0 , under varying environmental demands.

The simulation results for agent 0 are summarised in table 2 . For the sake of simplicity we consider only user requests arriving at agent 0 . Requests from other agents are processed in a similar way except for the fact that a conflict between a user request and a request from some other agent is always resolved in favour of the user request.

We now describe the entries in table 2 . Column 1 indicates the goal for which a request has been made. Column 2 gives the time at which the request arrived. Column 3 shows the deadline associated with the request. Columns 4 and 9 indicate the total processing time for the requested goal and the overhead incurred in processing that goal respectively. There are three possible ways in which a goal can be processed. The first possibility is for agent 0 itself to process the goal. In this case the total processing time is equal to the time indicated in table $1(T p)$ for that goal. No overhead is involved here. The second possibility arises when some already existing agent is given the contract for the goal as a result of coalition formation. In this case the total processing time is equal to the time

Table 1. Processing time and funds for goals.

\begin{tabular}{cccccc}
\hline Goal & Proc. time $(T p)$ & Funds & & \\
\hline$g 1$ & 10 & 100 & & \\
$g 2$ & 15 & 150 & & \\
$g 3$ & 12 & 120 & & \\
$g 4$ & 15 & 150 & $T d=2.0$ & \\
$g 5$ & 05 & 050 & & \\
$g 6$ & 05 & 050 & & \\
$g 7$ & 10 & 080 & & \\
$g 8$ & 09 & 090 & & \\
$g 9$ & 14 & 140 & & \\
$g 10$ & 05 & 050 & & \\
\hline
\end{tabular}


Table 2. Requests arriving at agent 0 .

\begin{tabular}{lcccccccc}
\hline $\begin{array}{l}\text { Goal } \\
\text { request } \\
(1)\end{array}$ & $\begin{array}{c}\text { Time of } \\
\text { arrival } \\
(2)\end{array}$ & $\begin{array}{c}\text { Deadline } \\
(3)\end{array}$ & $\begin{array}{c}\text { Total } \\
\text { pr. time } \\
(4)\end{array}$ & $\begin{array}{c}\text { Agent } \\
(5)\end{array}$ & $\begin{array}{c}\text { Completion } \\
\text { time } \\
(6)\end{array}$ & $\begin{array}{c}\text { Final result } \\
\text { at agent 0 } \\
(7)\end{array}$ & $\begin{array}{c}\text { Decommit- } \\
\text { ment time } \\
(8)\end{array}$ & $\begin{array}{c}\text { Over- } \\
\text { head } \\
(9)\end{array}$ \\
\hline$g 1$ & 00 & 12 & 10 & 0 & 10 & 10 & - & nil \\
$g 3$ & 05 & 30 & 12 & 0 & 22 & 22 & - & nil \\
$g 4$ & 10 & 40 & 15 & 0 & 37 & 37 & - & nil \\
$g 5$ & 15 & 45 & 05 & 0 & 42 & 42 & - & nil \\
$g 6$ & 20 & 50 & 05 & 0 & 47 & 47 & - & nil \\
$g 7$ & 26 & 50 & 12.5 & 1 & 38 & 38.5 & - & 2.5 \\
$g 8$ & 27 & 48 & 11 & 1 & 47 & 47.5 & - & 2 \\
$g 9$ & 28 & 56 & 18 & 2 & 45.5 & 46 & 34 & 4 \\
$g 10$ & 30 & 55 & 05 & 0 & 52 & 52 & - & nil \\
$g 5$ & 32 & 48 & 09 & 3 & 40.5 & 41 & - & 4 \\
$g 5$ & 35 & 60 & 05 & 0 & 57 & 57 & - & nil \\
$g 9$ & 34 & 56 & 16 & 3 & 54.5 & 55 & - & 9 \\
\hline
\end{tabular}

required for coalition formation $(T c)+$ the time required to process the goal as given in table $1(T p)+$ the time required to obtain the results from the contractor agent $(T r)$. The overhead incurred is equal to $T c+T r$. The third possibility arises when an attempt for coalition formation with existing agents fails and a new agent is created via decomposition which then processes the request. In this case the total processing time is equal to the time spent in trying for coalition formation $(T c)+$ time required for decomposition $(T d)+$ time required to process the goal $(T p)+$ time required to obtain results from the newly created contractor agent $(T r)$. The overhead incurred is equal to $T c+T d+T r$. In case the contractor agent decommits a goal, the overhead also includes the time that the contractor agent actually spent in processing the goal before decommitting and the time spent in trying coalition formation the second time. In this example we assume the $T c, T d$ and $T r$ values to be $1.5,2.0$ and 0.5 respectively.

Entries in column 5 indicate the agent to which the contract for the goal is finally awarded. Column 6 indicates the time at which an agent completes a goal that was awarded to it. In column 7 the completion time of the goal, i.e. the time at which the final results of processing the requested goal become available at agent 0 is given. Each value in column 7 is equal to the sum of the corresponding value in column 6 and Tr. If an agent decommits a goal that was assigned to it, then column 8 gives the time at which the decommittment takes place.

The first request arrives at time 0 for $g 1$. Agent 0 starts processing it immediately and completes it at time $10(T p)$. The next request arrives at time 5 for $g 3$. But as agent 0 is busy with $g 1$ at time 5 , it only starts processing it at time 10 and completes it at time $10+T p=22$. This continues for the third, fourth and fifth requests and each of these is completed before its deadline. There is no overhead for any of these requests. However when the sixth request for $g 7$ is made at time 26 , agent 0 is busy processing $g 4$ and it still has pending requests for $g 5$ and $g 6$ which are in the queue at the time. Agent 0 becomes free only after completing $g 6$, i.e. at time 47 . If agent 0 were to start $g 7$ at time 47 it would complete it at time $47+T p=57$. This however crosses the deadline. Therefore agent 0 does a decomposition at time 26 and creates a new agent, agent 1 . The time required for decomposition Td is two units. Thus agent 1 comes into existence at time 28 , starts 
processing $g 7$ and completes it at time $28+T p=38$. It then communicates the result to agent 0 . Thus agent 0 obtains the result from agent 1 at time $38+\operatorname{Tr}=38.5$. The overhead for $g 7$ is $T d+T r=2.5$ time units.

The seventh request is made at time 27 for $g 8$. As agent 0 cannot meet its deadline, it forms a coalition and awards the contract for $g .8$ to agent 1 at time $27+T c=28.5$. Agent 1 starts its execution after completing its current goal $g 7$ at time 38 , completes $g 8$ at $38+T p=47$ and this result becomes available to agent 0 at time $47+T r=47.5$. The overhead for $g 8$ is $T c+T r=2$ units of time.

The eighth request is made at time 28 for $g 9$. Its deadline cannot be met by agent 0 . This leads to an attempt for coalition formation which fails as agent 1 also cannot meet its deadline. A new agent, agent 2, is created through decomposition and $g 9$ is assigned to it. Agent 2 therefore starts executing $g^{9}$ at time $28+T c+T d=31.5$ and is expected to complete it at time $31.5+T p=45.5$. The remaining three requests can be processed along the same lines.

At time 34, agent 0 receives a decommittment message from agent 2 for $g 9$. As agent 0 cannot meet its deadline, it forms a coalition and awards the contract to agent 3 . Agent 3 becomes free at time 40.5 when it starts $g 9$ and completes it at time $40.5+T p=54.5$. This result becomes available to agent 0 at time $54.5+T r=55$. The overhead for $g 9$ is the sum of the time spent in trying coalition formation and decomposition: Once at the time of its first arrival at 28 and once again when it is decommitted at time 34, and the time that agent 2 spends in executing $g 9$ before decommitting it and $T r$, i.e. $2 \mathrm{~T} c+2 \mathrm{~T} d+1.5+T r=9$ units of time.

In this example we let an agent perform composition if it remains free for 15 units of time.

From these figures the following conclusions can be drawn.

Adaptiveness of the organisation: We have shown that when the response time exceeds the time limit, the organisation starts decomposition. Similarly it can be seen that when the response time drops below the time limit the organisation starts composition. The organisation of the system thus varies with varying demands from the environment.

Time constrained problem solving: In the given example the number of agents in the system varies between 1 and 4 . It can be said that in order to meet the deadlines of all the requests, an average of around two agents are required. If the same experiment is done using the conventional approach with two permanent agents, then the deadlines of all the requests cannot be met. Thus the reorganisation approach is more effective for time-constrained problem-solving.

Effective resource utilisation: In order to meet the deadlines of all the requests the conventional approach would require 4 permanent agents. Thus the reorganisation-centred approach which requires around two agents on an average is more economical.

Communication and reorganisation overhead: The overhead indicated in column 9 of table 2 is the sum of the communication and reorganisation overheads. Reorganisation overhead does not pose a problem as it is a temporary overhead. Communication overhead will not be significant in message passing machines. However communication overhead can become a problem when using wide-area networks to perform distributed problemsolving. 


\section{Conclusion}

To overcome the drawbacks of the available organisational policies when used in isolation, we presented a protocol that integrates these approaches by exploiting the negotiation mechanism of the contract net protocol, social laws of the social reasoning mechanism, market economy feature of the distributed computational economy and organisation self design to meet the needs of time-constrained domains where the computational load on the organisation cannot be predicted ahead of time.

Our simulation study shows that the proposed model has the characteristics of adaptiveness, meeting deadlines and efficient resource utilisation. This study is preliminary and we propose to put this model in operation in more realistic problem domains and study in detail the issues like scalability and stability of the model.

\section{References}

Cammarata S, Mc Arthur D, Steeb R 1983 Strategies for co-operation in distributed problem solving. Proc. of Eighth International Conference on Artificial Intelligence August, pp 767770

Cheng G, Ishida T 1996 Analysing the social behaviour of contract net protocol, Lecture Notes in Artificial Intelligence, No. 1038 (Berlin: Springer) pp 116-127

Riecken D (ed.) 1994 Commun. ACM (Special Issue on Intelligent Agents) 37(7): 18-21

Davis R, Smith R G 1983 Negotiation as a metaphor for distributed problem solving. Artif. Intell. 20: $63-109$

Decker K S 1987 Distributed problem solving techniques: A survey. IEEE Trans. Syst., Man Cybern. 7: 729-740

Durfee E H, Lesser V R, Corkill D D1985 Coherent co-operation among communicating problem solvers. Tech Report 85-15, Dept. Comput. Inf. Sci., Univ. Massachusetts, Amherst, MA

Fatima S S, Uma G 1998 An adaptive organisational policy for multi-agent systems - AASMAn. Proc. IEEE Int. Conf. on MultiAgent Systems 98: 120-127

Ishida T 1995 Parallel, distributed and multi-agent production systems. Proc. Int. Conf. MultiAgent Systems-95, pp 416-422

Ishida T, Gasser L, Yokoo M 1992 Organisation self design of production systems. IEEE Trans. Knowledge Data Eng. 4: 123-134

Sichman J S, Demazeau Y 1995 Exploiting social reasoning to enhance adaptation in open multiagent systems. Proc. Int. Conf. MultiAgent Systems-95

Smith R G 1980 Contract net protocol: High level communication and control in a distributed problem solver. IEEE Trans. Comput. 29: 1104-1113

Smith R G, Davis R 1981 Frameworks for co-operation in distributed problem solvers. IEEE Trans. Syst. Man Cybern. 11: 66-70

Waldspurger C A, Hogg T, Huberman B A, Kephart J O, Stornetta W S 1992 Spawn: A distributed computational economy. IEEE Trans. Software Eng. 18: 103-117 